\title{
Cardiac Involvement in a Pediatric Patient with COVID-19: Looking Beyond the Nonspecific Global Cardiac Injury
}

\author{
Suneet Bhansali ${ }^{1}$, Prashant Minocha ${ }^{1}$, Colin Phoon $^{1}$, Gillian Henry ${ }^{1}$, Sujata Chakravarti ${ }^{1}$, \\ Michelle Ramirez ${ }^{1}$, and Puneet Bhatla ${ }^{1}$ \\ ${ }^{1}$ NYU Langone Medical Center
}

May 21, 2020

\begin{abstract}
We report a case of a 17-year-old healthy male presenting with multisystem hyperinflammatory shock temporally associated with COVID-19. Cardiac involvement was suspected based on evidence of significant cardiac injury (elevated cardiac biomarkers, electrocardiographic and echocardiographic abnormalities). Cardiac magnetic resonance imaging was performed demonstrating global biventricular systolic dysfunction, as well as a small area of T2 hyperintensity and mid wall late gadolinium enhancement. This case discusses the varied cardiac involvement in pediatric patients with COVID-19 infection and highlights that cardiac injury is not just limited to hyperinflammatory syndrome related global dysfunction but a more focal myocarditis can also be seen.
\end{abstract}

\section{Introduction:}

The highly infectious 2019 novel coronavirus disease (COVID-19) is currently devastating populations around the world, affecting patients of all ages. Commonly, this disease presentation ranges from mild flulike illnesses and pneumonia to severe acute respiratory distress syndrome and death. ${ }^{1-2}$ Cardiovascular involvement has been described in adults with direct and indirect cardiac injury. ${ }^{3-6}$ There have been several reports of myocarditis in the adult population. ${ }^{3,6}$ Most recently there have been reports of clusters of pediatric patients presenting with severe multisystem inflammatory disease related to COVID-19 infection. These patients were noted to have cardiac involvement including ventricular systolic dysfunction and coronary artery aneurysms. ${ }^{7}$ We herein describe a case of an adolescent male presenting with COVID-19 related hyperinflammatory shock syndrome with a combination of diffuse and focal cardiac involvement.

\section{Case Presentation:}

A 17-year-old male with no significant comorbidities presented to the emergency department with seven days of fever, abdominal pain, diarrhea, emesis, and neck pain. There was no reported cough, dyspnea, chest pain, palpitations or syncope. On presentation, he was febrile to $39.5^{\circ} \mathrm{C}$, tachycardic with a heart rate of $150 \mathrm{bpm}$, and hypotensive with blood pressure 79/66 mmHg. His oxygen saturation was greater than $96 \%$ in room air and he was in no distress. Heart and lung examinations were normal. He had diffuse abdominal tenderness without hepatomegaly.

Blood tests demonstrated elevated levels of markers of myocardial damage and inflammation, including serum troponin, N-terminal pro-brain natriuretic peptide (NT-proBNP), C-reactive protein, D-Dimer, and Interleukin-6. Other routine blood tests were performed (Table 1). 2019 Novel Coronavirus SARS-COV-2 was detected by nasopharyngeal swab polymerase chain reaction assay. Pertinent miscellaneous tests including urine Legionella antigen and gastrointestinal adenovirus, Ebstein-Barr virus, human herpesvirus-6, enterovirus, and cytomegalovirus yielded no positive results. 12-lead electrocardiogram showed normal sinus rhythm with T-wave inversions in the inferior leads (Figure 1). Cardiac point of care ultrasound (CPOCUS) 
showed left ventricular (LV) dysfunction. Chest radiograph revealed a normal cardio-thymic silhouette and no infiltrates or focal consolidations.

Three normal saline boluses were administered with improvement in tachycardia and hypotension. Broad spectrum antibiotics (piperacillin and tazobactam) were administered for presumed sepsis. The patient was transferred to the pediatric intensive care unit (PICU) for management of suspected hyperinflammatory syndrome related to cardiac dysfunction and shock.

Upon admission to the PICU, another CPOCUS study was performed using a Philips Lumify transducer which showed mildly decreased LV systolic function (LVEF of $43 \%$ by $5 / 6$ area length method) and regional wall motion abnormalities predominantly involving the mid-ventricular level inferior wall as well as the inferior septum (Video 1). Cardiac magnetic resonance imaging (CMRI) was performed to further evaluate myocardial function and characterization; this confirmed the diminished LVEF of $40 \%$ and regional hypokinesia, but also showed mild systolic dysfunction of the right ventricle (RVEF of 39\%). Additionally, CMRI showed a small area of mid wall late gadolinium enhancement (LGE) involving the mid-ventricular level inferior septum at the inferior LV-RV junction (Figure 2a). This corresponded with the area of increased myocardial signal on T2-weighted fast spin echo images (Figure 2b), suggestive of myocardial edema. Overall findings were consistent with global cardiac dysfunction but also confirmed focal inflammation of the myocardium. Hydroxychloroquine was initiated empirically for COVID-19 once the QTc was confirmed to be normal but was discontinued on day 3 given QTc prolongation. He remained hemodynamically stable during his hospitalization without the use of vasopressors. His electrocardiographic abnormalities resolved, and troponin levels that peaked at $6.170 \mathrm{ng} / \mathrm{mL}$, were near-normal by hospital day 5 (Table 1). He was discharged home on day 5 of hospitalization with outpatient follow-up by cardiology.

\section{Discussion and review of literature:}

The 2019 novel coronavirus disease is caused by the SARS-CoV-2. The virus replicates in symptomatic and asymptomatic people and has resulted in more than 323,000 deaths worldwide as of May 20th, 2020 (https://coronavirus.jhu.edu/map.html). Disease presentation varies from nonspecific symptoms (fever, cough, sore throat, malaise, abdominal pain, diarrhea, vomiting) ${ }^{1,7}$ to pneumonia and acute respiratory distress syndrome (ARDS). ${ }^{2}$ Cardiovascular involvement is increasingly recognized in critically ill adult patients; the spectrum varies from myocarditis to acute heart failure/cardiomyopathy, and may increase the risk of acute myocardial infarction. ${ }^{4}$ In adults indirect cardiac injury related to a hyperinflammatory process and cytokine storm has been noted. ${ }^{8}$

On April $27^{\text {th }} 2020$, the Paediatric Intensive Care Society released a statement describing a rise in critically ill pediatric patients presenting with severe multisystem inflammatory disease related to COVID-19 infection. A recently published report, ${ }^{7}$ together with numerous anecdotal reports from Spain, France, the United Kingdom and the Unites states have described patients presenting with COVID-19 related hyperinflammatory shock syndrome. These patients had a range of clinical presentations including classic Kawasaki disease, atypical Kawasaki disease and toxic shock syndrome, with and without cardiac dysfunction. The mechanism of the cardiac injury is unclear but may be associated with a dramatic inflammatory response and cytokine storm. ${ }^{7,8}$ The clinical presentations reported are very similar to our patient, with unrelenting fever, severe gastrointestinal symptoms, hypotension, tachycardia, and minimal to no respiratory involvement. Elevated markers of cardiac injury have also been commonly reported in these patients pointing towards a more global cardiac involvement as part of the hyperinflammatory shock syndrome. ${ }^{7}$ None of these reports in children however describe concomitant focal myocarditis. Our case presented with focal electrocardiographic abnormalities (T-wave inversion in inferior leads) as well as regional wall motion abnormalities on echocardiography prompting further investigation for other potential mechanisms of cardiac involvement, such as inflammation.

Inflammation of cardiac muscle, or myocarditis, may be due to direct virus-related injury to cardiomyocytes or as a consequence of indirect injury from systemic inflammatory response syndrome or ARDS-induced hypoxemia. ${ }^{9}$ Currently, CMRI is the non-invasive gold standard to detect myocardial dysfunction, edema, 
and fibrosis, and to diagnose myocarditis. ${ }^{10}$ Since its emergence in December 2019, there have been few reported cases of COVID-19 related myocarditis in adults ${ }^{6}$, but none in children. Due to focal electrocardiographic abnormalities, regional dysfunction on echocardiography, and reports of varying cardiovascular involvement in adults with COVID-19, we obtained a CMRI to further delineate the mechanism of cardiovascular involvement in our patient. CMRI confirmed a diagnosis of focal myocarditis (suggested by T2 hyper-intensity and mid-wall LGE) apart from a previously well-described phenomenon of global cardiac dysfunction related to hyperinflammatory syndrome. In addition, CMRI provided other useful information in terms of diagnosing RV dysfunction (not well visualized by Lumify), and ruling out acute coronary syndrome, reported in adult patients. ${ }^{6}$ In contrast to coronary involvement in children with multisystem inflammatory disease related to COVID-19 infection, ${ }^{7}$ our patient had no coronary involvement, but rather cardiac dysfunction and features consistent with focal myocarditis.

\section{Conclusion:}

This is the first reported pediatric case of COVID-19 related hyperinflammatory shock with cardiac dysfunction and associated focal myocarditis, suspected based on elevated cardiac-injury-related biomarkers, electrocardiographic and echocardiographic abnormalities, and confirmed using CMRI. A high index of suspicion for myocardial involvement should be maintained in children with COVID-19. Focal myocarditis can be concomitantly seen in these pediatric patients who present with hyperinflammatory syndrome related global cardiac dysfunction. If logistically feasible, CMRI should be considered in these pediatric patients as it may provide significant insights into possible mechanisms of cardiovascular involvement and allow for improved risk stratification.

\section{Contributors' Statement:}

Suneet Bhansali and Prashant Minocha gathered and reviewed all data, designed, drafted, and revised the final manuscript. Gillian Henry, Sujata Chakravarti and Michelle Ramirez gathered and analyzed the clinical and imaging data, and contributed to drafting and editing the manuscript. Colin Phoon reviewed the clinical data, case management, electrocardiographic and echocardiographic data, and revised the final manuscript. Puneet Bhatla acquired and interpreted the echocardiographic and MRI images, and critically revised the final report. All authors approved the final manuscript as submitted and agree to be accountable for all aspects of the work in ensuring that questions related to the accuracy or integrity of any part of the work are appropriately investigated and resolved.

\section{References:}

1. Velavan TP, Meyer CG: The COVID-19 epidemic. Trop Med Int Health 2020:25(3): 278-280.

2. Kannan S, Shaik S, Ali P, et al: COVID-19 (Novel Coronavirus 2019) - recent trends. Eur Rev Med Pharmacol Sci 2020:24(4):2006-2011.

3. Zheng YY, Ma YT, Zhang JY, et al: COVID-19 and the cardiovascular system. Nat Rev Cardiol 2020:17(5):259-260.

4. Li B, Yang J, Zhao F, Zhi L, et al: Prevalence and impact of cardiovascular metabolic diseases on COVID-19 in China. Clin Res Cardiol 2020:109(5):531-538.

5. Bansal M. Cardiovascular disease and COVID-19. Diabetes Metab Syndr Clin Res Rev 2020:14(3):247250.

6. Hendren NS, Drazner MH, Bozkurt B, et al: Description and proposed management of the Acute COVID-19 Cardiovascular Syndrome. Circulation. Published online April 16, 2020.

7. Riphagen S, Gomez X, Gonzalez-Martinez C, et al: Hyperinflammatory shock in children during COVID-19 pandemic. Lancet. Published online May 6, 2020.

8. Mehta P, McAuley DF, Brown M, et al: COVID-19: consider cytokine storm syndromes and immunosuppression. Lancet 2020:395(10229):1033-1034.

9. Tunuguntla H, Jeewa A, Denfield S. Acute myocarditis and pericarditis in children. Pediatr Rev: 2019:40(1):14-25.

10. Friedrich M, Sechtem U, Schulz-Menger J, et al: International consensus group on cardiovascular ma- 
gnetic resonance in myocarditis. cardiovascular magnetic resonance in myocarditis: a JACC White Paper. J Am Coll Cardiol 2009:53(17):1475-87.

\section{Table 1: Clinical Laboratory Values}

\begin{tabular}{llll}
\hline Clinical Laboratory Values & Clinical Laboratory Values & Clinical Laboratory Values & Clinical Laboratory \\
\hline Test & Units & Reference Range & Emergency Departme \\
Troponin I & $\mathrm{ng} / \mathrm{mL}$ & $<0.04$ & 2.97 \\
B-type natriuretic peptide & $\mathrm{pg} / \mathrm{mL}$ & $<100$ & 2124 \\
N-terminal pro-brain natriuretic peptide & $\mathrm{pg} / \mathrm{mL}$ & $5-125$ & \\
Creatinine Kinase Total & $\mathrm{U} / \mathrm{L}$ & $29-168$ & 1218 \\
D-Dimer & $\mathrm{ng} / \mathrm{mL} \mathrm{DDU}$ & $<230$ & 382 \\
Lactate dehydrogenase & $\mathrm{IU} / \mathrm{L}$ & $84-246$ & 167 \\
C-Reactive Protein & $\mathrm{mg} / \mathrm{L}$ & $0-5$ & 1274.6 \\
Ferritin & $\mathrm{ng} / \mathrm{mL}$ & $8-388$ & 28 \\
Interleukin-6 & $\mathrm{pg} / \mathrm{mL}$ & $<5$ & 52 \\
Aspartate aminotransferase & $\mathrm{IU} / \mathrm{L}$ & $5-34$ & 44 \\
Alanine aminotransferase & $\mathrm{IU} / \mathrm{L}$ & $0-50$ & 128 \\
Sodium & $\mathrm{mmol} / \mathrm{L}$ & $136-145$ & 3.4 \\
Potassium & $\mathrm{mmol} / \mathrm{L}$ & $3.5-4.8$ & 8.7 \\
Calcium & $\mathrm{mg} / \mathrm{dL}$ & $8.8-10.4$ & 1.25 \\
Creatinine & $\mathrm{mg} / \mathrm{dL}$ & $0.7-1.3$ & 8.6 \\
White blood cell count & $10^{3} / \mathrm{uL}$ & $3.8-9.8$ & 80 \\
Neutrophil & $\%$ & $33-75$ & 11 \\
Lymphocyte & $\%$ & $16-53$ & \\
\hline
\end{tabular}

\section{Figure Legend:}

Table 1: Clinical Laboratory Values

Figure 1: Admission 12 lead electrocardiogram with sinus tachycardia and T-wave inversion in inferior leads

Figure 2a: Phase sensitive inversion recovery (PSIR) sequences in short axis view shows mid wall late gadolinium enhancement involving the inferior septum at the midventricular level (annotated by arrow).

Figure 2b: Turbo inversion recovery (TIRM) sequences in short axis view shows small area of increased signal in the myocardium involving the inferior septum at the midventricular level corresponding to the area of mid wall late gadolinium enhancement (see Figure 2a) suggestive of myocardial edema.

Video 1: An echocardiogram in parasternal short axis view showing mid-ventricular regional wall dysfunction. 


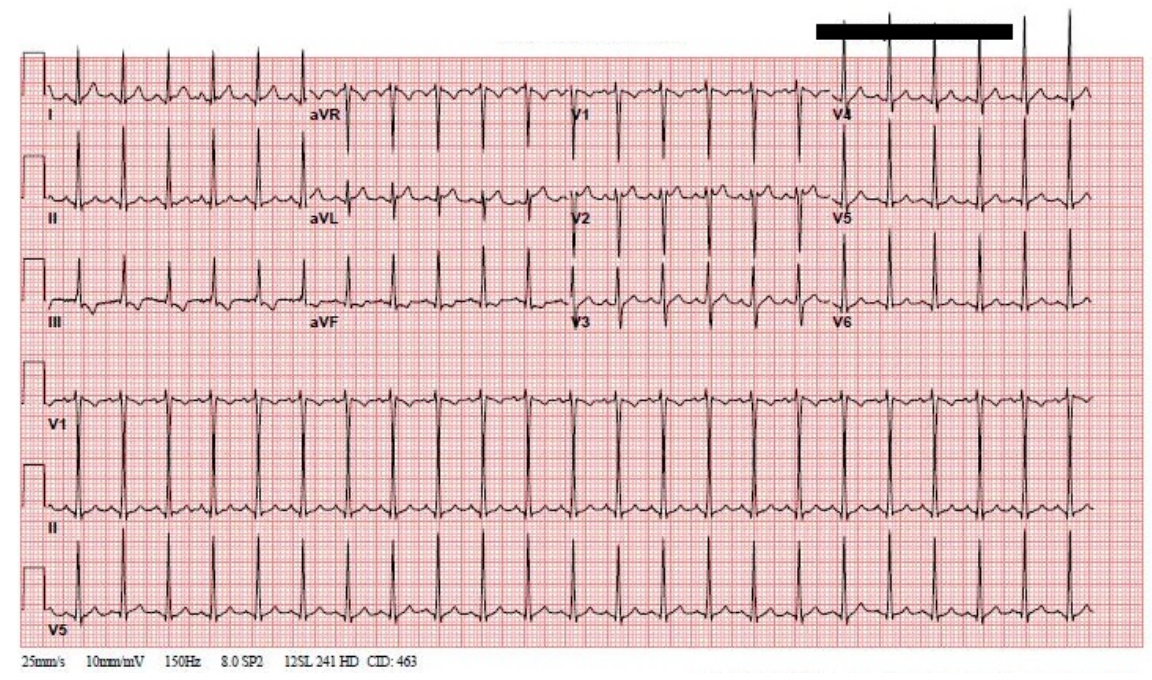




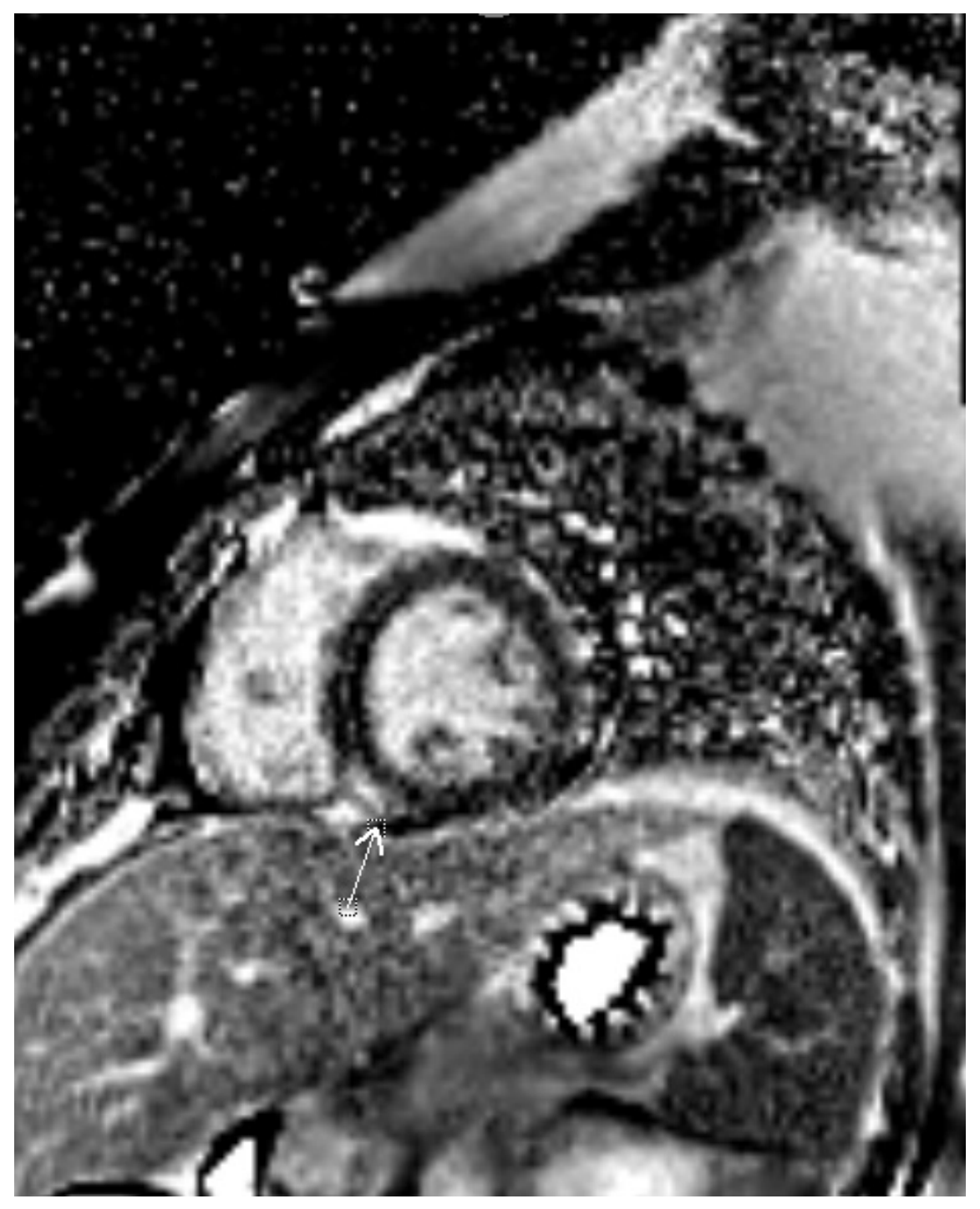




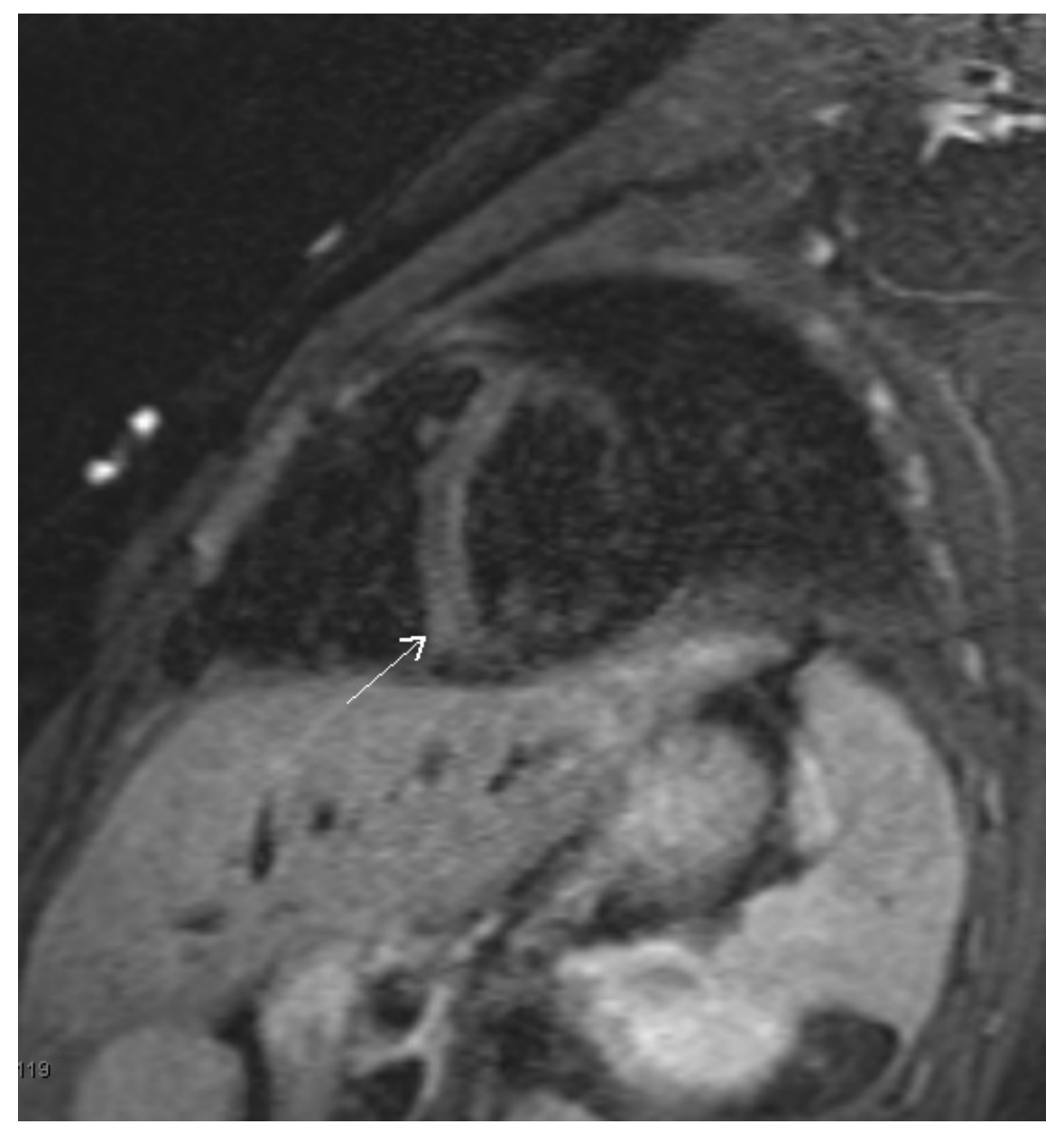

